\title{
Comentarios
}

\section{El método de la historización de los conceptos de Ignacio Ellacuría}

En nuestra realidad es usual la utilización de conceptos como los de justicia, libertad, democracia, desarrollo económico, etc., que tienen gran incidencia en la vida diaria de nuestra gente. El problema es que muchos de esos conceptos que se proclaman como buenos para todos, conllevan una serie de racionalizaciones que buscan justificar la situación de injusticia y de pobreza que afecta a la mayoría y legitimar la opulencia de unos pocos. ¿Cómo hacer para desenmasacarar ese uso falsificado de la razón?

La historización de los conceptos de Ignacio Ellacuría es un método con el potencial suficiente para realizar esta urgente tarea. El método de la historización es fruto de comprender la historia como apropiación y actualización de posiblidades y no como un mero despliegue de potencias que, desde el principio, están ya contenidas en la naturaleza, en el hombre o en un espíritu absoluto. Precisamente, el entender lo más radicalmente histórico de la historia hizo que Ellacuría combatiera presupuestos filosóficos de la historia que, en sus consecuencias reales, siempre condenan a las grandes mayorías empobrecidas. "Este es el sentido fundamental" —dice Antonio González-“del recurso de Ellacuría a una filosofía que, como la de Zubiri, pone en cuestión las categorías fundamentales que han determinado el pensamiento occidental desde los griegos hasta la modernidad" ("Filosofía de la historia y liberación", en Voluntad de vida. Ensayos filosóficos, Managua, 1993, p. 112). Ellacuría aplicó la concepción histórica zubiriana, a través del método de la historización, a conceptos como el de los derechos humanos, la propiedad y el bien común, los cuales se proclaman como verdaderos sin tener en cuenta las condiciones a las que se aplican, y que, muchas veces, ocasionan resultados muy contradictorios a los que dicen pretender.

El objetivo de este breve trabajo no es aplicar el método de la historización a un concepto determinado. Lo que buscamos es, en base a las aplicaciones hechas por Ellacuría identificar las principales características de este método para que pueda servir de matriz para la historización de muchos de los conceptos que tanto hace falta cuestionar e historizar en nuestra realidad. Para ello, nos hemos basado en tres escritos de Ignacio Ellacuría, "La historización del concepto de propiedad como principio de desideologización", en $E C A$, 1976, 335-336, pp. 425-450; "La historización del bien común y de los derechos humanos en una sociedad dividida" en Elsa Tames y Saúl Trinidad, Capitalismo: violencia y anti-vida, Costa Rica, 1978, pp. 81-94 y "La historización de los derechos humanos desde los pueblos oprimidos y las mayorías populares", en $E C A, 1990,502$, pp. 589-596.

La historización supone que los conceptos tienen que ver no con abstracciones, sino con realidades. Partamos entonces de lo que Ellacuría entiende por concepto histórico. Concepto histórico 


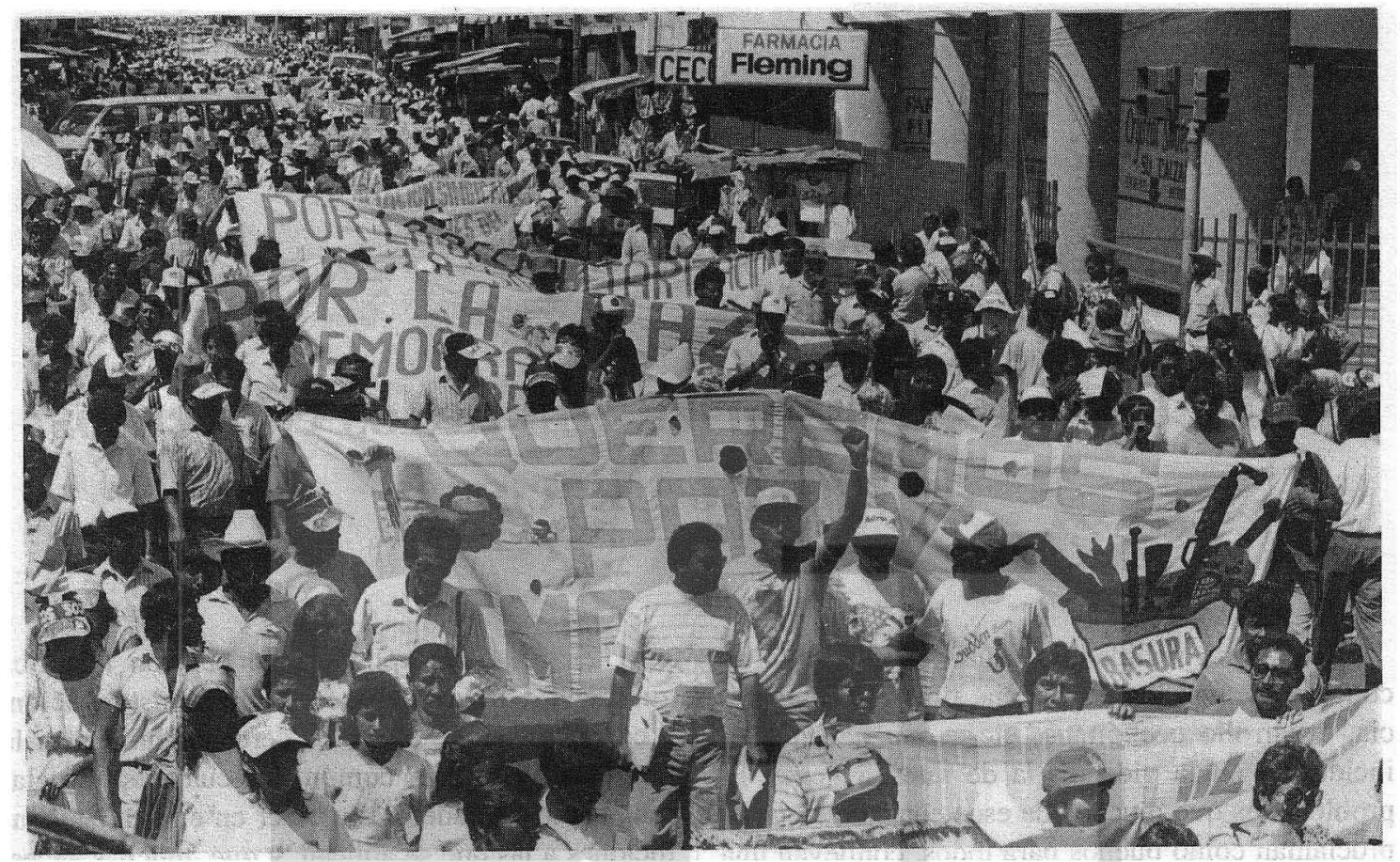

es lo opuesto al concepto abstracto o universal. El concepto universal es aquel que tiene la pretención de ser verdadero en todo lugar y en todo tiempo. Precisamente al hablar de conceptos históricos, referidos a una situación concreta en la que surgen, estamos hablando de conceptos que cobran diversos significados según sea la situación en la que se proclaman como verdaderos. Ellacuría los define como "conceptos operativos, cuya verdad se puede medir en sus resultados y cuyo contenido debe ir cambiando aunque se siga manteniendo su sentido esencial" ("La historización del concepto de propiedad como principio de desideologización", p. 591). No se trata de tirar por la borda la universalidad de los conceptos, sino de que el contenido de dichas formulaciones debe ir cambiando conforme cambia la realidad a la que se aplican. Por tanto, un concepto histórico es aquel que responde, en sus contenidos, a la realidad histórica. La verdad de los conceptos históricos no está tanto en su formulación teórica, sino en su realización práctica.

Tener claro lo que significan los conceptos históricos es de suma importancia para desenmascarar lo que encubren los conceptos abstractos que no tienen correlato en la realidad. Para Ellacuría, las ideologías dominantes viven de una falacia fundamental: "la de dar como conceptos reales e históricos, como valores efectivos y operantes, como pautas de acción eficaces, unos conceptos o representaciones, unos valores y unas pautas de acción, que son abstractos y universales" (Ibid.). El método de la historización de los conceptos surge, entonces, como necesidad para hacer históricos unos conceptos abstractos y universales que, en el fondo, lo que hacen es salvaguardar los intereses de los sectores privilegiados de una sociedad.

Ignacio Ellacuría, en sus artículos, va explicitando progresivamente lo que entiende por historización. En "La historización del concepto de propiedad como principio de desideologización", el primero de sus artículos sobre el tema, escrito en 1976, enfoca el método, básicamente, como principio para desenmasacarar la racionalización falsificada de los conceptos que lo que hacen es encubrir y legitimar el orden establecido. La historización es entonces principio de desideologización. Según el padre Ellacuría, la historización consistiría en "mostrar qué van dan- 
do de sí en una determinada realidad ciertos conceptos" (Ibid.).

En su segundo artículo, que data de 1978, "La historización del bien común y de los derechos humanos en una sociedad dividida", manteniendo la dimensión desideologizadora del método, Ellacuría utiliza la historización de los conceptos como principio de verificación. La historización consiste, en primer lugar, "en ver cómo se está realizando en una circunstancia dada lo que se afirma abstractamente... y, en segundo lugar, en la posición de aquellas condiciones reales sin las que no se puede dar la realización efectiva" (p. 88) de los conceptos en cuestión. De lo que se trata entonces es, por un lado, de verificar, en la realidad concreta, si lo que se pretende realizar en el concepto se cumple y, por otro, de identificar los aspectos que obstaculizan o viabilizan ese cumplimiento.

Ya cercana la fecha de su martirio, en mayo de 1989, Ellacuría escribió su tercer artículo, "La historización de los derechos humanos desde los pueblos oprimidos y las mayorías populares". En él, expone más sistemáticamente este método como lo veremos más adelante. Por ahora lo que nos interesa es definir qué se entiende aquí por historización. En este tercer artículo, además de presentar la historización como principio de desideologización y de verificación, introduce la dimensión tiempo "para poder cuantificar y verificar cuando las proclamaciones ideales se pueden convertir en realidades o alcanzar, al menos, cierto grado aceptable de realización" (p. 591).

Como puede verse, lo que se entiende por historización ha tenido una especie de evolución en los escritos de Ellacuría, quien fue explicitando progresivamente en qué consiste su método.

Recogiendo los planteamientos en los tres artículos, nos atrevemos a sostener que la historización de los conceptos consiste en verificar si en una realidad determinada se da lo que formalmente se presenta en el concepto, en descubrir si lo que hace el concepto en esa realidad determinada está al servicio de los intereses de los grupos privilegiados que son precisamente los que más reivindican dicho concepto, en identificar cuáles son las condiciones que impiden la realización efectiva del concepto y cuáles son las que pueden poner en marcha el proceso de esa realización y en cuantificar el tiempo prudencial para constatar un grado aceptable de cumplimiento de lo planteado en el concepto como un "deber ser".

Definido en qué consiste la historización de los conceptos, veamos brevemente algunas de las características de este método.

Es innegable que muchos conceptos aplicados a la realidad cotidiana de la gente, están impregnados de racionalizaciones que falsifican la realidad. Si esto es así, ¿de dónde surgen las ideologizaciones? El problema es que el ser humano no ocupa su falcultad cognitiva sólo para conocer lo que lo rodea, sino que, esencialmente, para hacer frente a la supervivencia. De ahí que todo conocimiento sea interesado; se trata de un interés por la vida que limita la posibilidad para llegar a la objetividad. El problema se agrava cuando entran en juego intereses mayores que afectan a individuos y a grupos en sociedades, como las del llamado tercer mundo, donde existe una marcada división entre los pocos que viven en la opulencia y los muchos

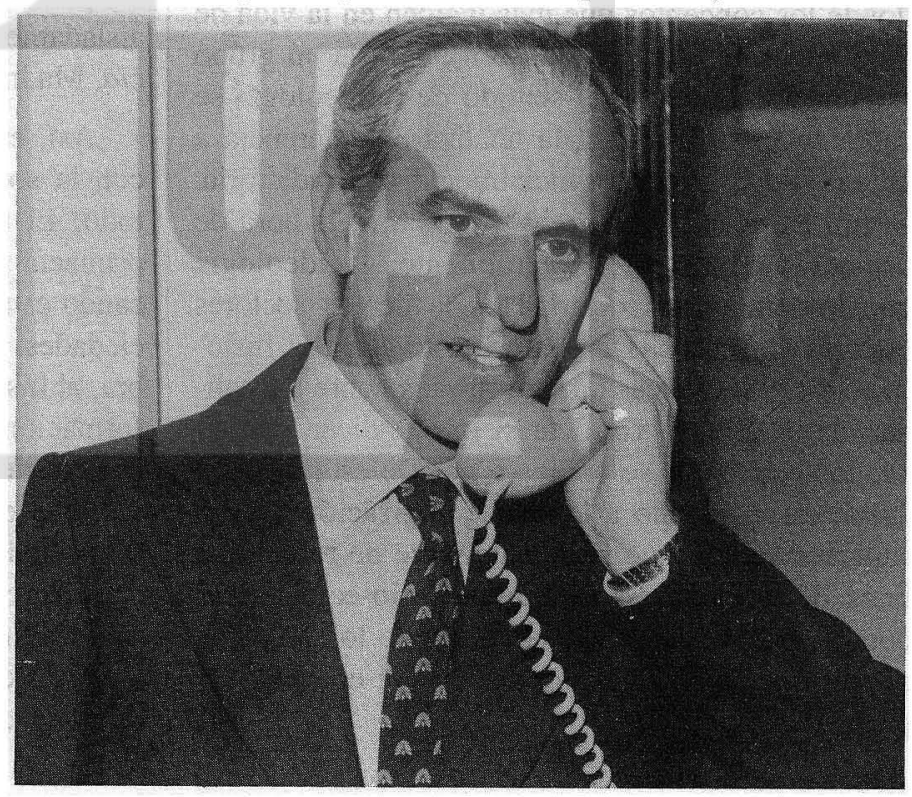


que viven en la indigencia. En defensa de esos determinados intereses surgen las racionalizaciones, es decir, un ejercicio falsificado de la razón que busca defender esas posiciones y no la objetividad del conocimiento. Es aquí donde radica el carácter ideologizado de los conceptos.

Por consiguiente, en la historización entendemos las ideologías "como racionalización encubridora y falsificada de intereses reales" ("Historización del concepto de propiedad", p. 587), es decir, como algo que "siempre hace referencia a la totalidad del pensamiento, a un conjunto de representaciones y valoraciones de la realidad social que procuran o pretenden ocultar determinadas relaciones sociales y legitimar determinada realidad" (Carlos Ayala y Henry A. Avilés, "Historización del concepto de democracia en los partidos políticos: PCN, PDC, ARENA", Tesis de licenciatura, UCA, junio de 1985, p. 14).

Así, la estructura ideológica de un determinado sistema económico-político se presenta como un marco teórico que hace aparecer la realidad legitimada como la mejor forma de organización posible y aceptable para los hombres, como la más racional y en consonancia con las leyes naturales y divinas. Por medio de los conceptos ideologizados se persigue defender un determinado sistema económico-político. En las sociedades divididas, donde los conceptos que más inciden en la vida de la gente están ideologizados en favor de un grúpo minúsculo, es donde el sentido de la ideología se capta mejor, porque se la analiza en referencia a una realidad social esencialmente contradictoria. Según Ellacuría, no hay que olvidar, a la hora de desideologizar, que, generalmente, la ideología que impera en la sociedad es la de los sectores más privilegiados. De lo que se trata en la historización es de volcar nuestra mirada sobre la realidad de la gente y verificar si lo que dicen pretender los conceptos se realiza o no. De esta manera, "estamos ayudando a la desideologización de una superestructura, que realmente sirve de refuerzo a unas determinadas estructuras socio-económicas que, de hecho, causan lo contrario de lo que dicen querer operar" ("La historización del concepto de propiedad", p. 592).
En sociedades divididas como las de nuestra América Latina, caracterizadas por el deterioro en las condiciones de vida de la mayoría de la población, es urgente tomar posición. Aquí se propone enfocar la historización "no sólo desde la perspectiva del triunfo de la razón sobre la fuerza sino, más en concreto, desde la defensa del débil en contra del fuerte" ("Historización de los derechos humanos", p. 590). En realidad, Ellacuría no elaboró - no lo dejaron hacerlo- de una manera filosófica y sistemática, el "desde" de su historización. No obstante, en sus escritos hay material para intentar una fundamentación filosófica de por qué el "desde las mayorías" es el enfoque más correcto para el método de la historización.

En primer lugar, en todo pensamiento de tipo estructural -como los de Aristóteles, Santo Tomás, Hegel, Marx, Zubiri- la unidad es anterior a las partes y tiene primacía sobre ellas. Como diría Ellacuría, aunque esto pudiera parecer "totalitario", es una idea sustentada muy explícitamente. Para Zubiri, que la unidad sea anterior a las partes es algo innegable: "Primaria significa que, sea cualquiera el mecanismo de su producción, en la unidad, una vez existente, cada nota es función de las demás, de suerte que sólo en y por su unidad con las restantes es cada nota lo que es dentro de la cosa real. En este sentido, la unidad domina, es un prius respecto de la posición de cada nota aisladamente considerada" (Zubiri, Sobre la esencia, Madrid, 1962, p. 143).

Así, en nuestro caso, la relación del individuo con la sociedad es la relación de la parte con el todo. El todo de la sociedad es anterior y tiene primacía sobre cada uno de los individuos. Aplicando esta consideración estructural a nuestras sociedades, la perspectiva "desde las mayorías" cobra, al historizar, un potencial de denuncia y transformación importante. Para Ellacuría, en El Salvador, por ejemplo, lo que sucede es "la anulación del todo estructural en beneficio de algunas partes disgregadoras de ese todo..." ("La historización del bien común y de los derechos humanos en una sociedad dividida", p. 85), y, más en general, "en una estructura capitalista burguesa, es claro el predominio de la parte sobre el todo y también de la 
parte menor sobre la parte mayor" (p. 91). Por el bienestar del todo de la sociedad, debemos tomar partido por la mayoría de la gente y no por la parte, minúscula, pero disgregadora.

En segundo lugar y apoyados en el argumento anterior, apelamos a la epistemología. El ubicarnos "desde" las mayorías implica que hemos optado por el que creemos es el mejor lugar para conocer. Ya dijimos que la raíz de las ideologizaciones se encuentra en el condicionamiento de la lucha por la supervivencia individual y grupal. Ese condicionamiento es el que nos aleja de un conocimiento veraz. En nuestras sociedades - no hay que olvidarlo-, la conciencia colectiva está configurada por los intereses de la clase dominante ("La historización del concepto de propiedad", pp. 589-590). La ideologización de los conceptos se da, en la mayoría de los casos, a favor de los sectores privilegiados. Entonces, desde el punto de vista de quien busca la verdad, la posición que defiende los derechos de la mayoría, está más cerca de esa verdad que la de quienes defienden los derechos de una minoría. En una sociedad contradictoria, en donde la mayoría sufre a causa de una minoría, es más probable que las razones esgrimidas por la mayoría tenga mayor validez - una validez ética y social - y que al ser sistematizadas adecuadamente se acerquen a la objetividad.

En efecto, para Ellacuría, "la verdad real de un proceso histórico está en los resultados objetivos de ese proceso... y más en concreto, la verdad real de un proceso histórico no está ni en los resultados obtenidos que deberían constituir el bien común, ni menos aún en las minorías que se apropian de esos resultados, sino en la participación de ese bien común y en el estado real de la mayoría de los hombres y de los ciudadanos" ("Historización del bien común").

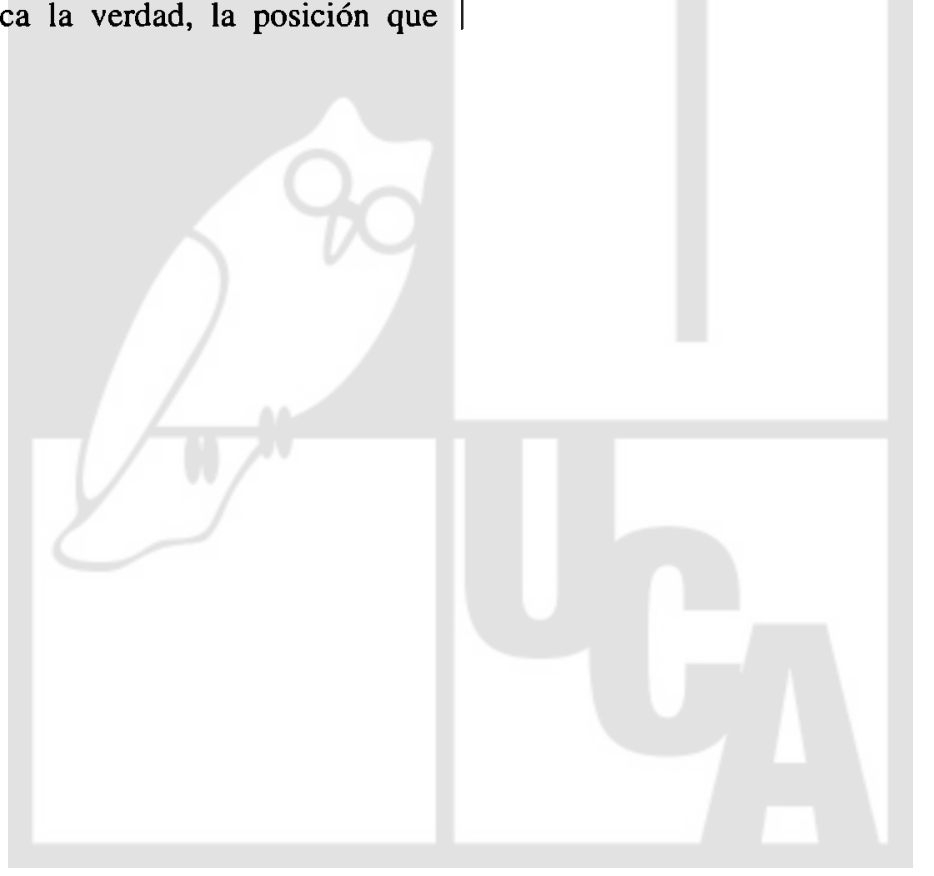

O. S. 